\title{
Closed form solutions for water-filling problems in optimization and game frameworks
}

\author{
Eitan Altman \\ INRIA BP93 \\ 2004 route des Lucioles \\ 06902 Sophia Antipolis \\ FRANCE \\ altman@sophia.inria.fr
}

\author{
Konstantin Avrachenkov \\ INRIA BP93 \\ 2004 route des Lucioles \\ 06902 Sophia Antipolis \\ FRANCE \\ k.avrachenkov@sophia.inria.fr
}

\author{
Andrey Garnaev \\ St. Petersburg State University \\ Universitetskii pr 35 \\ Peterhof \\ St Petersburg 198504 \\ RUSSIA \\ agarnaev@rambler.ru
}

\begin{abstract}
We study power control in optimization and game frameworks. In the optimization framework there is a single decision maker who assigns network resources and in the game framework players share the network resources according to Nash equilibrium. The solution of these problems is based on so-called water-filling technique, which in turn uses bisection method for solution of non-linear equations for Lagrange multiplies. Here we provide a closed form solution to the water-filling problem, which allows us to solve it in a finite number of operations. Also, we produce a closed form solution for the Nash equilibrium in symmetric Gaussian interference game. In addition, to its mathematical beauty, the explicit solution allows one to study limiting cases when the crosstalk coefficient is either small or large. We provide an alternative simple proof of the convergence of the Iterative Water Filling Algorithm. Furthermore, it turns out that the convergence of Iterative Water Filling Algorithm slows down when the crosstalk coefficient is large. Using the closed form solution, we can avoid this problem. Finally, we compare the non-cooperative approach with the cooperative approach and show that the non-cooperative approach results in a more fair resource distribution. ${ }^{1}$
\end{abstract}

\section{INTRODUCTION}

In wireless networks and DSL access networks the total available power for signal transmission has to be distributed among several resources. In the context of wireless networks, the resources may correspond to frequency bands (e.g. as in OFDM), or they may correspond to capacity available at different time slots. In the context of DSL access networks, the resources correspond to available frequency tones. This spectrum of problems can be considered in either optimiza-

\footnotetext{
${ }^{1}$ This work was partly supported by BioNets European project and by the joint RFBR and NNSF Grant no.0601-39005.
}

Permission to make digital or hard copies of all or part of this work for personal or classroom use is granted without fee provided that copies are not made or distributed for profit or commercial advantage and that copies bear this notice and the full citation on the first page. To copy otherwise, to republish, to post on servers or to redistribute to lists, requires prior specific permission and/or a fee.

GameComm '07, October 22, 2007, Nantes, France

Copyright 2007 ICST 978-963-9799-00-4. tion scenario or as a result of a non-cooperativie game scenario. The optimization scenario leads to "Water Filling Optimization Problem" $[4,6,13]$ and the game scenario leads to "Water Filling Game" or "Gaussian Interference Game" $[8,11,12,14]$. In the optimization scenario, one needs to maximize a concave function (Shannon capacity) subject to power constraints. The Lagrange multiplier corresponding to the power constraint is determined by a non-linear equation. In the previous works $[4,6,13]$, it was suggested to find the Lagrange multiplier by means of a bisection algorithm, where comes the name "Water Filling Problem". Here we show that the Lagrange multiplier and hence the optimal solution of the water filling problem can be found in explicit form with a finite number of operations. In the multiuser context, one can view the problem in either cooperative or non-cooperative setting. If a centralized controller wants to maximize the sum of all users' rates, the controller will face a non-convex optimization problem [3]. On the other hand, in the non-cooperative setting, the power allocation problem becomes a game problem where each user perceives the signals of the other users as interference and maximizes a concave function of the noise to interference ratio. In $[8,14]$ the spectrum of available resources was continuous, here as in $[10,11,12]$ we consider the discrete spectrum of available resources. A natural approach in the non-cooperative setting is the application of the Iterative Water Filling Algorithm (IWFA) [15]. Recently, the authors of [10] proved the convergence of IWFA under fairly general conditions. In the present work we consider the case of symmetric water filling game with two users. The restriction to the symmetric scenario allows us to find Nash equilibrium in explicit form. In addition, to its mathematical beauty, the explicit solution allows one to find the Nash equilibrium in water filling game in a finite number of operations and to study limiting cases when the crosstalk coefficient is either small or large. As a by-product, we obtain an alternative simple proof of the convergence of the Iterative Water Filling Algorithm. Furthermore, it turns out that the convergence of IWFA slows down when the crosstalk coefficient is large. Using the closed form solution, we can avoid this problem. Finally, we compare the non-cooperative approach with the cooperative approach and conclude that the cost of anarchy is small in the case of small crosstalk coefficients. We also show that the non-cooperative approach results in a more fair resource distribution. Applications that can mostly benefit from decentralized non-cooperative power control are ad-hoc and sensor 
networks with no predefined base stations [5, 9, 7]. An interested reader can find more references on non-cooperative power control in $[2,8]$. We would like to mention that the water filling problem and jamming games with transmission costs have been analyzed in [1].

The structure of the paper is as follows: In the next Section 2, we recall the single decision maker setup of the water filling optimization problem. Then, in Section 3 we provide its explicit solution. In Section 4 we formulate two user symmetric water filling game and characterize its Nash equilibrium. In Section 5 we give an alternative simple proof of the convergence of the iterative water filling algorithm. In Section 6 we give the explicit form of the players' strategy in the Nash equilibrium. In Section 7 we confirm our finding with the help of numerical example. In that section we also show that the cost of anarchy is small when the crosstalk coefficient is small. We make conclusions in Section 8.

\section{SINGLE DECISION MAKER}

We consider the following power allocation problem in the case of a single decision maker. There is a single decision maker (also called "Transmitter") who wants to send information using $n$ independent resources so as to maximize the Shannon capacity. We further assume that resource $i$ has a "weight" of $\pi_{i}$.

Possible interpretations:

(i) The resources may correspond to capacity available at different time slots; we assume that there is a varying environment whose state changes among a finite set of states $i \in[1, n]$, according to some ergodic stochastic process with stationary distribution $\left\{\pi_{i}\right\}_{i=1}^{n}$. We assume that both players have perfect knowledge of the environment state at the beginning of each time slot.

(ii) The resources may correspond to frequency bands (e.g. as in OFDM) where one should assign different power levels for different sub-carriers [13]. In that case we may take $\pi_{i}=1 / n$ for all $i$.

The strategy of Transmitter is $T=\left(T_{1}, \ldots, T_{n}\right)$ with $\sum_{i=1}^{n} \pi_{i} T_{i}=\bar{T}, T_{i} \geq 0, \pi_{i}>0$ for $i \in[1, n]$ and $\bar{T}>0$. As the payoff to Transmitter we take the Shannon capacity

$$
v(T)=\sum_{i=1}^{n} \pi_{i} \ln \left(1+\frac{T_{i}}{N_{i}^{0}}\right),
$$

where $N_{i}^{0}>0$ is the noise level in the sub-carrier $i$. Define also the function

$$
H_{T}(\omega)=\sum_{i=1}^{n} \pi_{i} T_{i}(\omega)
$$

We would like to emphasize that the above generalized description of the water-filling problem can be used for power allocation in time as well as power allocation in space-frequency. Following the standard water-filling approach $[4,6$, 13], which assumes application Kuhn-Tacker Theorem, we have the following result.

TheOREM 1. Let $T_{i}(\omega)=\left[1 / \omega-N_{i}^{0}\right]_{+}$for $i \in[1, n]$. Then $T\left(\omega^{*}\right)=\left(T_{1}\left(\omega^{*}\right), \ldots, T_{n}\left(\omega^{*}\right)\right)$ is the unique optimal strategy and its payoff is $v\left(T\left(\omega^{*}\right)\right)$ where $\omega^{*}$ is the unique root of the equation

$$
H_{T}(\omega)=\bar{T}
$$

We observe that the above result can be easily proved without application of the Kuhn-Tacker conditions. In the appendix we supply this proof.

\section{CLOSED FORM SOLUTION FOR WATER FILLING PROBLEM}

In the previous studies of the water-filling problems it was suggested to use numerical (e.g., bisection) method to solve equation (1). Here we propose an explicit form approach for the solution of equation (1).

Without loss of generality we can assume that the subcarriers are arranged by the noise level as follows:

$$
\frac{1}{N_{1}^{0}} \geq \frac{1}{N_{2}^{0}} \geq \ldots \geq \frac{1}{N_{n}^{0}}
$$

THEOREM 2. The solution of the water-filling optimization problem is given by

$$
T_{i}^{*}= \begin{cases}\frac{\bar{T}+\sum_{t=1}^{k} \pi_{t}\left(N_{t}^{0}-N_{i}^{0}\right)}{\sum_{t=1}^{k} \pi_{t}}, & \text { if } i \leq k, \\ 0, & \text { if } i>k,\end{cases}
$$

where $k$ can be found from the following conditions:

$$
\varphi_{k}<\bar{T} \leq \varphi_{k+1},
$$

where

$$
\varphi_{t}=\sum_{i=1}^{t} \pi_{i}\left(N_{t}^{0}-N_{i}^{0}\right) \text { for } t \in[1, n] .
$$

Let us demonstrate the closed form approach by a numerical example. Take $n=5, \bar{T}=1, N_{i}^{0}=\kappa^{i-1}, \kappa=1.7, \pi_{i}=1 / 5$ for $i \in[1,5]$. Then, as the first step we calculate $\varphi_{t}$ for $t \in[1,5]$. In our case we get (0. 0.14, 0.616, 1.8298, 4.58108). Then, by (3), $k=3$. Thus, by (2), the optimal water-filling strategy is $T^{*}=(2.53,1.83,0.64,0,0)$ with payoff 0.438 .

\section{SYMMETRIC WATER FILLING GAME}

Two Transmitters try to send information through $n$ resources so as to maximize the quality of the transmitted information. The strategy of Transmitter $j$ is $T^{j}=\left(T_{1}^{j}, \ldots, T_{n}^{j}\right)$ with $T_{i}^{j} \geq 0$ and with

$$
\sum_{i=1}^{n} \pi_{i} T_{i}^{j}=\bar{T}^{j}
$$

where $\bar{T}^{j}>0$ for $j=1,2$. The payoffs to Transmitters are given as follows

$$
\begin{aligned}
& v^{1}\left(T^{1}, T^{2}\right)=\sum_{i=1}^{n} \pi_{i} \ln \left(1+\frac{T_{i}^{1}}{g T_{i}^{2}+N_{i}^{0}}\right), \\
& v^{2}\left(T^{1}, T^{2}\right)=\sum_{i=1}^{n} \pi_{i} \ln \left(1+\frac{T_{i}^{2}}{g T_{i}^{1}+N_{i}^{0}}\right),
\end{aligned}
$$

where $g \in(0,1)$. These payoffs correspond to Shannon capacities. This is an instance of the Water-Filling or Gaussian Interference Game $[8,14,15]$. In the important particular cases of OFDM wireless network and DSL access network, 
$\pi_{i}=1 / n, i=1, \ldots, n$. In this work we restrict ourselves to the case of symmetric game with equal crosstalk coefficients. This situation can correspond for example to the scenario when the transmitters are situated at about the same distance from the base station.

We shall characterize a Nash Equilibrium of this problem. The strategies $\left(T^{1 *}, T^{2 *}\right)$ constitute a Nash Equilibrium, if for any strategies $\left(T^{1}, T^{2}\right)$ the following inequalities hold:

$$
\begin{aligned}
& v^{1}\left(T^{1}, T^{2 *}\right) \leq v^{1}\left(T^{1 *}, T^{2 *}\right), \\
& v^{2}\left(T^{1 *}, T^{2}\right) \leq v^{2}\left(T^{1 *}, T^{2 *}\right) .
\end{aligned}
$$

Since $v^{1}$ and $v^{2}$ are concave in $T^{1}$ and $T^{2}$ respectively, the Kuhn-Tucker conditions imply the following theorem.

THEOREM 3. $\left(T^{1 *}, T^{2 *}\right)$ is a Nash equilibrium if and only if there are non-negative $\omega^{1}$ and $\omega^{2}$ (Lagrange multipliers) such that

$$
\frac{1}{g T_{i}^{m *}+T_{i}^{j *}+N_{i}^{0}} \begin{cases}=\omega^{j} & \text { for } T_{i}^{j *}>0, \\ \leq \omega^{j} & \text { for } T_{i}^{j *}=0,\end{cases}
$$

where $j, m \in\{1,2\}$ and $m \neq j$.

We would like to emphasize that the positivity of the Lagrange multipliers $\omega^{1}$ and $\omega^{2}$ is specific for our problem. In general, the Lagrange multipliers corresponding to equality constrants do not have any restrictions on the sign.

Let us introduce the following sets:

$$
\begin{aligned}
& I_{00}^{\prime}\left(\omega^{1}, \omega^{2}\right)=\left\{i \in[1, n]: \frac{1}{\omega^{1}} \leq N_{i}^{0}, \quad \frac{1}{\omega^{2}} \leq N_{i}^{0}\right\} \\
& I_{10}^{\prime}\left(\omega^{1}, \omega^{2}\right)=\left\{i \in[1, n]: N_{i}^{0}<\frac{1}{\omega^{1}} \text { and either } N_{i}^{0} \geq \frac{1}{\omega^{2}}\right. \\
&\text { or } \left.N_{i}^{0}<\frac{1}{\omega^{2}} \text { and } \frac{1}{\omega^{2}}-N_{i}^{0} \leq g\left(\frac{1}{\omega^{1}}-N_{i}^{0}\right)\right\}, \\
& I_{01}^{\prime}\left(\omega^{1}, \omega^{2}\right)=\left\{i \in[1, n]: N_{i}^{0}<\frac{1}{\omega^{2}} \text { and either } N_{i}^{0} \geq \frac{1}{\omega^{1}}\right. \\
&\text { or } \left.N_{i}^{0}<\frac{1}{\omega^{1}} \text { and } \frac{1}{\omega^{1}}-N_{i}^{0} \leq g\left(\frac{1}{\omega^{2}}-N_{i}^{0}\right)\right\}, \\
& I_{11}^{\prime}\left(\omega^{1}, \omega^{2}\right)=\{i \in[1, n]: \\
&\left.0<g\left(\frac{1}{\omega^{2}}-N_{i}^{0}\right)<\frac{1}{\omega^{1}}-N_{i}^{0}<\frac{1}{g}\left(\frac{1}{\omega^{2}}-N_{i}^{0}\right)\right\} .
\end{aligned}
$$

The next result characterizes the forms that the Nash equilibrium can take.

Lemma 1. Let $\left(T^{1 *}, T^{2 *}\right)$ be a Nash equilibrium, then

(i) if $T_{i}^{1 *}=0$ and $T_{i}^{2 *}=0$ then $i \in I_{00}^{\prime}\left(\omega^{1}, \omega^{2}\right)$,

(ii) if $T_{i}^{1 *}>0$ and $T_{i}^{2 *}=0$ then $i \in I_{10}^{\prime}\left(\omega^{1}, \omega^{2}\right)$ and

$$
T_{i}^{1 *}=1 / \omega^{1}-N_{i}^{0}
$$

(iii) if $T_{i}^{1 *}=0$ and $T_{i}^{2 *}>0$ then $i \in I_{01}^{\prime}\left(\omega^{1}, \omega^{2}\right)$ and

$$
T_{i}^{2 *}=1 / \omega^{2}-N_{i}^{0}
$$

(iv) if $T_{i}^{1 *}>0$ and $T_{i}^{2 *}>0$ then $i \in I_{11}^{\prime}\left(\omega^{1}, \omega^{2}\right)$ and

$$
\begin{aligned}
& T_{i}^{1 *}=\frac{\left(1 / \omega^{1}-N_{i}^{0}\right)-g\left(1 / \omega^{2}-N_{i}^{0}\right)}{1-g^{2}}, \\
& T_{i}^{2 *}=\frac{\left(1 / \omega^{2}-N_{i}^{0}\right)-g\left(1 / \omega^{1}-N_{i}^{0}\right)}{1-g^{2}} .
\end{aligned}
$$

Although the game has symmetric nature there are some non-symmetric features impacted by the fact that the Lagrange multiplies are different as a rule. This difference will allow us to simplify the structure of the sets $I^{\prime}$ and the strategies. For this purpose first introduce the following auxiliary notations for positive $\omega^{1}$ and $\omega^{2}$ :

(i) if $\omega^{1}<\omega^{2}$, so $1 / \omega^{2}<1 / \omega^{1}$ then let

$$
\begin{aligned}
& I_{00}\left(\omega^{1}, \omega^{2}\right)=\left\{i \in[1, n]: \frac{1}{\omega^{1}} \leq N_{i}^{0}\right\}, \\
& I_{10}\left(\omega^{1}, \omega^{2}\right)=\left\{i \in[1, n]: \frac{1 / \omega^{2}-g / \omega^{1}}{1-g} \leq N_{i}^{0}<\frac{1}{\omega^{1}}\right\}, \\
& I_{01}\left(\omega^{1}, \omega^{2}\right)=\emptyset \\
& I_{11}\left(\omega^{1}, \omega^{2}\right)=\left\{i \in[1, n]: N_{i}^{0}<\frac{1 / \omega^{2}-g / \omega^{1}}{1-g}\right\},
\end{aligned}
$$

(ii) if $\omega^{2}<\omega^{1}$, so $1 / \omega^{1}<1 / \omega^{2}$ then let

$$
\begin{aligned}
& I_{00}\left(\omega^{1}, \omega^{2}\right)=\left\{i \in[1, n]: \frac{1}{\omega^{2}} \leq N_{i}^{0}\right\}, \\
& I_{10}\left(\omega^{1}, \omega^{2}\right)=\emptyset, \\
& I_{01}\left(\omega^{1}, \omega^{2}\right)=\left\{i \in[1, n]: \frac{1 / \omega^{1}-g / \omega^{2}}{1-g} \leq N_{i}^{0}<\frac{1}{\omega^{2}}\right\}, \\
& I_{11}\left(\omega^{1}, \omega^{2}\right)=\left\{i \in[1, n]: N_{i}^{0}<\frac{1 / \omega^{1}-g / \omega^{2}}{1-g}\right\},
\end{aligned}
$$

(iii) if $\omega^{2}=\omega^{1}$ then let

$$
\begin{aligned}
& I_{00}\left(\omega^{1}, \omega^{2}\right)=\left\{i \in[1, n]: \frac{1}{\omega^{2}} \leq N_{i}^{0}\right\}, \\
& I_{10}\left(\omega^{1}, \omega^{2}\right)=\emptyset, \quad I_{01}\left(\omega^{1}, \omega^{2}\right)=\emptyset, \\
& I_{11}\left(\omega^{1}, \omega^{2}\right)=\left\{i \in[1, n]: N_{i}^{0}<\frac{1}{\omega^{2}}\right\} .
\end{aligned}
$$

The next lemma asserts that the sets $I^{\prime}$ are coincides with the sets $I$.

LEMMA 2. There are the following relations between sets $I^{\prime}$ and $I: I_{00}^{\prime}\left(\omega^{1}, \omega^{2}\right)=I_{00}\left(\omega^{1}, \omega^{2}\right), I_{10}^{\prime}\left(\omega^{1}, \omega^{2}\right)=I_{10}\left(\omega^{1}, \omega^{2}\right)$, $I_{01}^{\prime}\left(\omega^{1}, \omega^{2}\right)=I_{01}\left(\omega^{1}, \omega^{2}\right)$ and $I_{11}^{\prime}\left(\omega^{1}, \omega^{2}\right)=I_{11}\left(\omega^{1}, \omega^{2}\right)$ for positive $\omega^{1}$ and $\omega^{2}$.

Now we introduce some strategies, which the Nash Equilibrium will have the form of. Namely, for positive $\omega^{1}$ and $\omega^{2}$ such that $\omega^{1} \leq \omega^{2}$ and for $i \in[1, n]$ we introduce the following notations:

$$
\begin{aligned}
& T_{i}^{1}\left(\omega^{1}, \omega^{2}\right)= \\
& \begin{cases}\frac{1}{1+g}\left(\frac{\frac{1}{\omega^{1}}-\frac{g}{\omega^{2}}}{1-g}-N_{i}^{0}\right) & \text { if } N_{i}^{0}<\frac{\frac{1}{\omega^{2}}-\frac{g}{\omega^{1}}}{1-g}, \\
\frac{1}{\omega^{1}}-N_{i}^{0} & \text { if } \frac{\frac{1}{\omega^{2}}-\frac{g}{\omega^{1}}}{1-g} \leq N_{i}^{0}<\frac{1}{\omega^{1}}, \\
0 & \text { if } \frac{1}{\omega^{1}} \leq N_{i}^{0},\end{cases} \\
& T_{i}^{2}\left(\omega^{1}, \omega^{2}\right)= \\
& \begin{cases}\frac{1}{1+g}\left(\frac{\frac{1}{\omega^{2}}-\frac{g}{\omega^{1}}}{1-g}-N_{i}^{0}\right) \quad & \text { if } N_{i}^{0}<\frac{1 / \omega^{2}-g / \omega^{1}}{1-g} \\
0 & \text { if } \frac{\frac{1}{\omega^{2}}-\frac{g}{\omega^{1}}}{1-g} \leq N_{i}^{0}\end{cases}
\end{aligned}
$$


either in the following equivalent form as follows

$$
\begin{aligned}
& T_{i}^{2}\left(\omega^{1}, \omega^{2}\right) \\
& = \begin{cases}\frac{1}{1+g}\left(t^{2}-N_{i}^{0}\right) & \text { if } N_{i}^{0}<t^{2}, \\
0 & \text { if } t^{2} \leq N_{i}^{0},\end{cases} \\
& T_{i}^{1}\left(\omega^{1}, \omega^{2}\right) \\
& = \begin{cases}\frac{1}{1+g}\left((1+g) t^{1}-g t^{2}-N_{i}^{0}\right) & \text { if } N_{i}^{0}<t^{2}, \\
t^{1}-N_{i}^{0} & \text { if } t^{2} \leq N_{i}^{0}<t^{1}, \\
0 & \text { if } t^{1} \leq N_{i}^{0},\end{cases}
\end{aligned}
$$

where

$$
t^{2}=\frac{1 / \omega^{2}-g / \omega^{1}}{1-g}, \quad t^{1}=\frac{1}{\omega^{1}} .
$$

It is clear that

$$
1 / \omega^{1}=t^{2}, \quad 1 / \omega^{2}=g t^{1}+(1-g) t^{2} .
$$

For the case $\omega^{1}>\omega^{2}, T_{i}^{1}\left(\omega^{1}, \omega^{2}\right)$ and $T_{i}^{2}\left(\omega^{1}, \omega^{2}\right)$ can be defined by symmetry.

The next result simplifies the form of the Nash equilibrium, given by Lemma 1 and it shows that the strategies are not so symmetric as it could be expected and their nonsymmetric structure motivated by difference in Lagrange multipliers and so in the power of the signals the players have to transfer.

THEOREM 4. Each Nash equilibrium is of the form $\left(T^{1}\left(\omega^{1}, \omega^{2}\right), T^{2}\left(\omega^{1}, \omega^{2}\right)\right)$ for some positive $\omega^{1}$ and $\omega^{2}$.

The next result shows that there is a monotonous dependence between the power of the signals the players have to transfer and Lagrange multipliers.

Corollary 1. Let $\left(T^{1}\left(\omega^{1}, \omega^{2}\right), T^{2}\left(\omega^{1}, \omega^{2}\right)\right)$ be a Nash equilibrium. If $\bar{T}^{1}>\bar{T}^{2}$, then $\omega^{1}<\omega^{2}$.

To find the equilibrium strategies we have to find $\omega^{1}$ and $\omega^{2}$ such that the following conditions hold

$$
H^{j}\left(\omega^{1}, \omega^{2}\right)=\bar{T}^{j} \text { for } j=1,2
$$

where

$$
H^{j}\left(\omega^{1}, \omega^{2}\right)=\sum_{i=1}^{n} \pi_{i} T_{i}^{j}\left(\omega^{1}, \omega^{2}\right) \text { for } j=1,2 .
$$

The next Lemma shows that this system has the unique solution and moreover its proof supplies a simple method of its determination.

LEMMA 3. The system of non-linear equations (9) has unique positive solution $\left(\omega_{*}^{1}, \omega_{*}^{2}\right)$.

Proof. Without lost of generality we can assume that $\bar{T}^{1} \geq \bar{T}^{2}$. Let $\left(\omega^{1}, \omega^{2}\right)$ be the positive solution of (9). Then, by Corollary $1, \omega^{1}<\omega^{2}$. Thus, instead of the system of equation (9) with variables $\omega^{1}$ and $\omega^{2}$ we can consider the following equivalent system of equation (10) with variables $t^{1}$ and $t^{2}$ where $0<t^{2} \leq t^{1}$ :

$$
\tilde{H}^{2}\left(t^{2}\right)=\bar{T}^{2}, \quad \tilde{H}^{1}\left(t^{1}, t^{2}\right)=\bar{T}^{1},
$$

where

$$
\begin{gathered}
\tilde{H}^{2}\left(t^{2}\right)=\frac{1}{1+g} \sum_{\left\{i: t^{2}>N_{i}^{0}\right\}} \pi_{i}\left(t^{2}-N_{i}^{0}\right), \\
\tilde{H}^{1}\left(t^{1}, t^{2}\right)=\sum_{\left\{i: t^{2} \leq N_{i}^{0}<t^{1}\right\}} \pi_{i}\left(t^{1}-N_{i}^{0}\right) \\
+\sum_{\left\{i: N_{i}^{0}<t^{2}\right\}} \pi_{i} \frac{1}{1+g}\left((1+g) t^{1}-g t^{2}-N_{i}^{0}\right) .
\end{gathered}
$$

It is clear that $\tilde{H}^{2}(\cdot)$ is continuous in $(0, \infty), \tilde{H}^{2}(\tau)=0$ for $\tau \leq N_{1}^{0}, \tilde{H}^{2}(+\infty)=+\infty$ and $\tilde{H}^{2}(\cdot)$ is strictly increasing in $\left(N_{1}^{0}, \infty\right)$. Then, there is the unique positive $t_{*}^{2}$ such that

$$
\tilde{H}^{2}\left(t_{*}^{2}\right)=\bar{T}^{2} .
$$

It is clear that $\tilde{H}^{1}\left(\cdot, t_{*}^{2}\right)$ is continuous and increasing in $\left(t_{*}^{2}, \infty\right), \tilde{H}^{1}\left(\infty, t_{*}^{2}\right)=+\infty$ and $\tilde{H}^{1}\left(t_{*}^{2}, t_{*}^{2}\right)=\tilde{H}^{2}\left(t_{*}^{2}\right)=\bar{T}^{2} \leq$ $\bar{T}^{1}$. So, there is the unique positive $t_{*}^{1}$ such that

$$
\tilde{H}^{1}\left(t_{*}^{1}, t_{*}^{2}\right)=\bar{T}^{1} .
$$

So, the system (10) has the unique solution $\left(t_{*}^{1}, t_{*}^{2}\right)$. Thus, (9) also has the unique solution and it can be found by (8). This completes the proof of Lemma 3.

Lemmas 1-3 imply the following main result.

THEOREM 5. The symmetric water filling game has the unique Nash equilibrium $\left(T^{1}\left(\omega_{*}^{1}, \omega_{*}^{2}\right), T^{2}\left(\omega_{*}^{1}, \omega_{*}^{2}\right)\right)$ for $g \in$ $(0,1)$, where $\omega_{*}^{1}, \omega_{*}^{2}$ can be fount through $t_{*}^{1}$ and $t_{*}^{2}$ from (7) which are the unique solution of the triangular system of equations (10).

The assumption that $g<1$ is essential for the uniqueness of Nash Equilibrium as it is shown in the following Proposition.

Proposition 1. For $g=1$ the symmetric water filling game has a continuum of Nash equilibria.

\section{CONVERGENCE OF AN IWFA}

In this section we describe a version of the water-filling algorithm for finding the Nash Equilibrium and supply a simple proof of its convergence based on some monotonisity properties. It is clear that $H^{i}\left(\omega^{1}, \omega^{2}\right) i=1,2$ have the following properties, collected in the next Lemma, which follow directly from the explicit formulas of the Nash Equilibrium.

LEMMA 4. (i) $H^{i}\left(\omega^{1}, \omega^{2}\right), i=1,2$ are nonnegative and continuous, (ii) $H^{i}\left(\omega^{1}, \omega^{2}\right)$ is non-increasing on $\omega^{i}$, (iii) $H^{i}\left(\omega^{1}, \omega^{2}\right) \rightarrow \infty$ for $\omega^{i} \rightarrow 0$, (iv) $H^{i}\left(\omega^{1}, \omega^{2}\right)=0$ for enough big $\omega^{i}$, say for $\omega^{i} \geq 1 / N_{1}^{0}$, (v) $H^{i}\left(\omega^{1}, \omega^{2}\right)$ is non-decreasing by $\omega^{j}(j \neq i)$.

These properties give a simple proof of the convergence of the following water-filling algorithm for finding the Nash Equilibrium.

Let $\omega_{0}^{1}$ and $\omega_{0}^{2}$ be such that $H^{1}\left(\omega_{0}^{1}, \omega_{0}^{2}\right)=H^{2}\left(\omega_{0}^{1}, \omega_{0}^{2}\right)=0$, for example $\omega_{0}^{1}=\omega_{2}^{0}=1 / N_{1}^{0}$. Let $\omega_{1}^{2}=\omega_{0}^{2}$ and define $\omega_{1}^{1}$ such that $H^{1}\left(\omega_{1}^{1}, \omega_{1}^{2}\right)=\bar{T}^{1}$. Such $\omega_{1}^{1}$ exists by Lemma 4(i)(iii). Then, by Lemma $4(\mathrm{i}),(\mathrm{v}) H^{2}\left(\omega_{1}^{1}, \omega_{1}^{2}\right)=0$. Let $\omega_{2}^{1}=$ $\omega_{1}^{1}$ and define $\omega_{2}^{2}$ such that $H^{2}\left(\omega_{2}^{1}, \omega_{2}^{2}\right)=\bar{T}^{2}$. Then, by Lemma $4(\mathrm{v}) H^{1}\left(\omega_{2}^{1}, \omega_{2}^{2}\right) \leq \bar{T}^{1}$ and so on. So we have nonincreasing positive sequence $\left(\omega_{k}^{1}, \omega_{k}^{2}\right)$. Thus, it converges to an $\left(\omega_{*}^{1}, \omega_{*}^{2}\right)$ which produces a Nash Equilibrium. 


\section{CLOSED FORM SOLUTION FOR SYM- METRIC WATER FILLING GAME}

In this section, based on the proof of Lemma 3, we propose the solution of the two players symmetric water filling game in the closed form.

Without lost of generality we can assume that

$$
\bar{T}_{1}>\bar{T}_{2} \text {. }
$$

Let $k_{2}$ be such that $N_{k_{2}+1}^{0} \geq t_{*}^{2}>N_{k_{2}}^{0}$. Then, since $\tilde{H}^{2}\left(t_{*}^{2}\right)=$ $\bar{T}^{2}$, we have that

$$
t_{*}^{2}=\frac{(1+g) \bar{T}^{2}+\sum_{i=1}^{k_{2}} \pi_{i} N_{i}^{0}}{\sum_{i=1}^{k_{2}} \pi_{i}} .
$$

Since $\tilde{H}^{2}(\cdot)$ is strictly increasing, $k_{2}$ can be found from the condition

$$
\tilde{H}^{2}\left(N_{k^{2}}^{0}\right)<\bar{T}^{2} \leq \tilde{H}^{2}\left(N_{k^{2}+1}^{0}\right) .
$$

Hence, $k^{2}$ can be found from the following equivalent conditions:

$$
\varphi_{k^{2}}^{2}<\bar{T}^{2} \leq \varphi_{k^{2}+1}^{2}
$$

where

$$
\varphi_{k}^{2}=\frac{1}{1+g} \sum_{i=1}^{k} \pi_{i}\left(N_{k}^{0}-N_{i}^{0}\right),
$$

for $k \leq n$, and $\varphi_{n+1}^{2}=\infty$.

Since $t_{*}^{1}$ is the root of the equation $\tilde{H}^{1}\left(\cdot, t_{*}^{2}\right)=\bar{T}^{1}$ there is $k^{1} \geq k^{2}$ such that $N_{k^{1}+1}^{0} \geq t_{*}^{1}>N_{k^{1}}^{0}$. So,

(i) if $k^{1}>k^{2}$ then

$$
t_{*}^{1}=\frac{\bar{T}^{1}+\sum_{i=k^{2}+1}^{k^{1}} \pi_{i} N_{i}^{0}+\frac{1}{1+g} \sum_{i=1}^{k^{2}} \pi_{i}\left(g t_{2}^{*}+N_{i}^{0}\right)}{\sum_{i=1}^{k^{1}} \pi_{i}},
$$

(ii) if $k^{1}=k^{2}$ then

$$
t_{*}^{1}=\frac{\bar{T}^{1}+\frac{1}{1+g} \sum_{i=1}^{k^{2}} \pi_{i}\left(g t_{2}^{*}+N_{i}^{0}\right)}{\sum_{i=1}^{k^{1}} \pi_{i}} .
$$

Thus, $k^{1} \geq k^{2}$ can be found as follows:

(i) $k^{1}=k^{2}$ if $\bar{T}^{1} \leq \varphi_{k^{2}+1}^{1}$,

(ii) otherwise $k^{1}$ is given by the condition:

$$
\varphi_{k^{1}}^{1}<\bar{T}^{1} \leq \varphi_{k^{1}+1}^{1}
$$

where

$$
\begin{aligned}
\varphi_{k}^{1} & =\sum_{i=k^{2}+1}^{k} \pi_{i}\left(N_{k}^{0}-N_{i}^{0}\right) \\
& +\frac{1}{1+g} \sum_{i=1}^{k^{2}} \pi_{i}\left((1+g) N_{k}^{0}-N_{i}^{0}-g t_{*}^{2}\right)
\end{aligned}
$$

for $k \in\left[k_{2}+1, n\right]$, and $\varphi_{n+1}^{1}=\infty$.

We can summarize the obtained results in the following theorem.
Theorem 6. Let $\bar{T}_{1}>\bar{T}_{2}$. Then, the Nash equilibrium strategies are given by

$$
\begin{aligned}
& T_{i}^{1 *}= \begin{cases}t_{*}^{1}-\frac{g t_{*}^{2}+N_{i}^{0}}{1+g} & \text { if } i \in\left[1, k^{2}\right], \\
t_{*}^{1}-N_{i}^{0} & \text { if } i \in\left[k^{2}+1, k^{1}\right], \\
0 & \text { if } i \in\left[k^{1}+1, n\right],\end{cases} \\
& T_{i}^{2 *}= \begin{cases}\frac{1}{1+g}\left(t_{*}^{2}-N_{i}^{0}\right) & \text { if } i \in\left[1, k^{2}\right], \\
0 & \text { if } i \in\left[k^{2}+1, n\right],\end{cases}
\end{aligned}
$$

where $k^{2}, t_{*}^{2}, k^{1}$ and $t_{*}^{1}$ are given by (14), (13), (17) and (15).

\section{NUMERICAL EXAMPLE}

Let us demonstrate the closed form approach by a numerical example. Take $n=5, g=0.9, \bar{T}^{1}=5, \bar{T}^{2}=0.5, N_{i}^{0}=$ $\kappa^{i-1}, \kappa=1.7, \pi_{i}=1 / 5$ for $i \in[1,5]$. Then, as the first step we calculate $\varphi_{t}^{2}$ for $t \in[1,5]$. In our case we get $(0,0.074$, $0.324,0.963,2.411)$. Then, by $(14), k^{2}=3$. Thus, by (13) $t_{*}^{2}=3.447$. Then we calculate $\varphi_{t}^{1}$ for $t \in[4,5]$. In our case we get $(1.380,4.131)$. So, by $(17), k^{1}=5$. Using (15), we find $t_{*}^{1}=9.221$. Thus, by (18) we have the following equilibrium strategies $T^{1 *}=(7.062,6.694,6.067,4.308,0.869)$ and $T^{2 *}=(1.288,0.919,0.293,0,0)$ with payoffs 0.909 and 0.062 . We have run IWFA, which produced the same values for the optimal strategies and payoffs. However, we have observed that the convergence of IWFA is slow when $g \approx 1$. In Figure 1 we have plotted the total error in strategies $\left\|T_{k}^{1}-T^{1 *}\right\|_{2}+\left\|T_{k}^{2}-T^{2 *}\right\|_{2}$, where $T_{k}^{i}$ are the strategies produced by IWFA on the $k$-th iteration and $T^{i *}$ are the Nash equilibrium strategies. Our approach instantaneously finds the Nash equilibrium for all values of $g$. Also, it is interesting to note that by (18) the quantity of channels as well as the channels themselves used by weaker player (with smaller resources) is independent on behavior of the stronger player (with bigger resources) but of course each player allocating his/her resources among these channels take into account the opponent behaviour.

In Figure 2, we compare the non-cooperative approach with the cooperative approach. Specifically, we compare the transmission rates and their sum under Nash equilibrium strategies and under strategies obtained from the centralized optimization of the sum of transmitters' rates. The main conclusions are: the cost of anarchy is nearly zero for $g \in[0,1 / 4]$ and then it grows up to $22 \%$ when $g$ grows from $1 / 4$ to 1 ; the transmitter with more resources gains significantly more from the centralized optimization. Hence, the non-cooperative approach results in a more fair resource distribution.

In Table 1 we give strategies of both users obtained in the case of the centralized optimization for different values of the crosstalk coefficient $g$. First, we observe that when the crosstalk coefficient is large, the users occupy different resources. The user with the larger average power takes better resources. When the crosstalk coefficient is below 0.7 , the users start to share the resources. As the value of the crosstalk coefficient decreases, the 2nd user with the smaller average power begins to occupy better resources. As expected, when the crosstalk coefficient is very small, the optimal strategies start to look like strategies which are optimal in the case of no interference. 


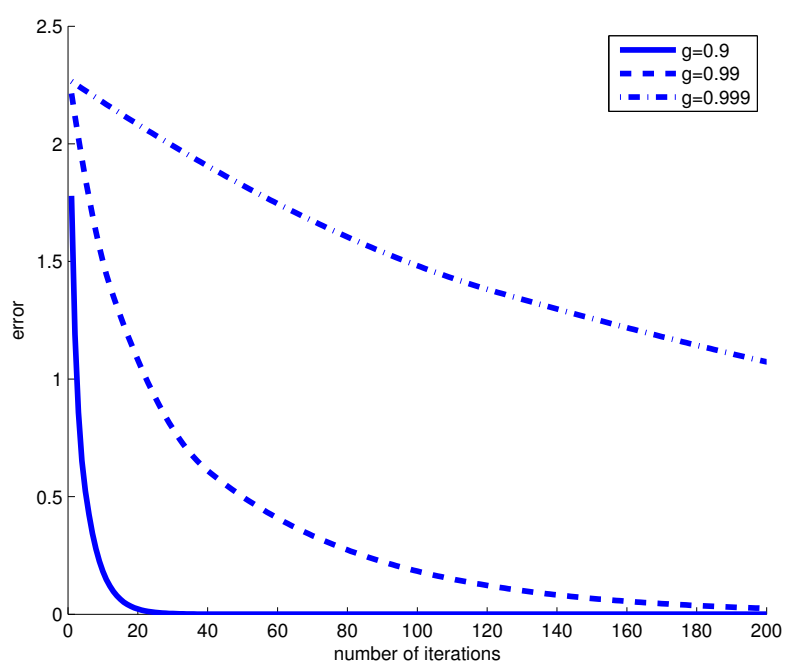

Figure 1: Convergence of IWFA

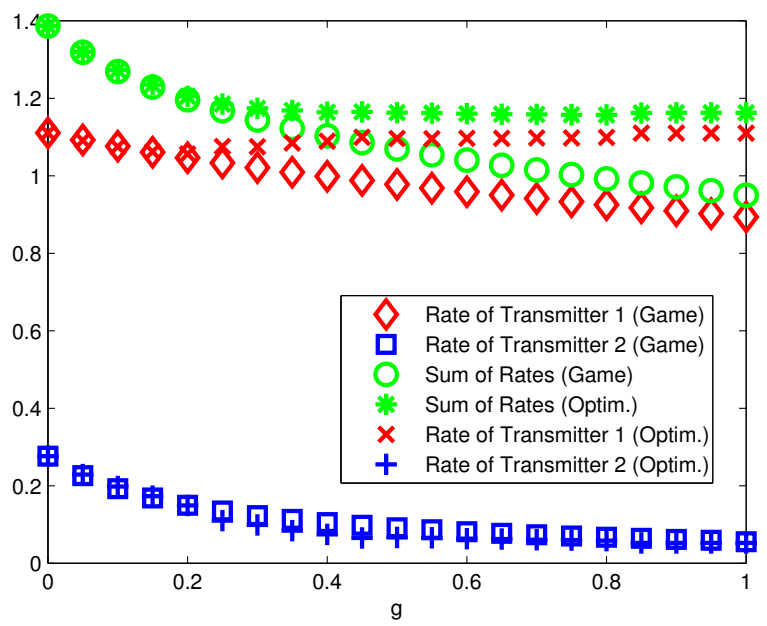

Figure 2: Centralized Optimization vs. Game

\section{CONCLUSION}

We have considered power control for wireless networks in optimization and game frameworks. Closed form solutions for the water filling optimization problem and two players symmetric water filling games have been provided. Namely, now one can calculate optimal/equilibrium strategies with a finite number of arithmetic operations. We have also provided a simple alternative proof of convergence for a version of iterative water filling algorithm. It had been known before that the iterative water filling algorithm converges very slow when the crosstalk coefficient is close to one. For our closed form approach possible proximity of the crosstalk coefficient to one is not a problem. We have shown that when the crosstalk coefficient is equal to one, there is a continuum of Nash equilibria. Finally, we have demonstarted that the price of anarchy is small when the crosstalk coefficient is small and that the decentralized solution is better than
Table 1: Centralized optimization

\begin{tabular}{lcccccc}
\hline \hline$g$ & & \multicolumn{5}{c}{ Users' strategies } \\
\hline \multirow{2}{*}{0.95} & 1st user & 7.43 & 7.17 & 6.19 & 4.19 & 0.00 \\
& 2nd user & 0.00 & 0.00 & 0.00 & 0.00 & 2.50 \\
0.70 & 1st user & 7.83 & 7.22 & 5.98 & 3.96 & 0.00 \\
& 2nd user & 0.00 & 0.00 & 0.00 & 0.00 & 2.50 \\
\multirow{2}{*}{0.65} & 1st user & 8.25 & 7.42 & 6.57 & 2.76 & 0.00 \\
& 2nd user & 0.00 & 0.00 & 0.00 & 0.83 & 1.67 \\
0.35 & 1st user & 8.27 & 7.73 & 4.77 & 3.33 & 0.90 \\
& 2nd user & 0.00 & 0.00 & 1.24 & 1.22 & 0.04 \\
0.20 & 1st user & 6.65 & 6.44 & 6.16 & 4.36 & 1.38 \\
& 2nd user & 0.91 & 0.99 & 0.60 & 0.00 & 0.00 \\
0.10 & 1st user & 6.27 & 6.65 & 6.47 & 4.53 & 1.08 \\
& 2nd user & 1.43 & 1.00 & 0.07 & 0.00 & 0.00 \\
\multirow{2}{*}{0.01} & 1st user & 7.45 & 7.03 & 6.00 & 3.98 & 0.54 \\
& 2nd user & 1.58 & 0.92 & 0.00 & 0.00 & 0.00 \\
\hline
\end{tabular}

centralized with respect to fairness.

We expect that the present approach can be generalized to the case of more than two users. A detail analytical study of the centralized optimization is another future research topic.

\section{APPENDIX}

Proof of Theorem 1. Let $T^{*}=\left(T_{1}^{*}, \ldots, T_{n}^{*}\right)$ be the optimal strategy. Since $\sum_{i=1}^{n} \pi_{i} T_{i}^{*}=\bar{T}$ and $T_{i}^{*} \geq 0$ for $i \in[1, n]$ there is a $m \in[1, n]$ such that $T_{m}^{*}>0$.

Let $\epsilon$ be any small enough positive number and $k \neq m$. Let $T^{\epsilon, k}=\left(T_{1}^{\epsilon, k}, \ldots, T_{n}^{\epsilon, k}\right)$ be such that

$$
T_{i}^{\epsilon, k}= \begin{cases}T_{m}^{*}-\epsilon / \pi_{m} & \text { for } i=m, \\ T_{k}^{*}+\epsilon / \pi_{k} & \text { for } i=k, \\ T_{i}^{*} & \text { for } i \notin\{m, k\} .\end{cases}
$$

It is cleat that $T^{\epsilon, k}$ also is a strategy for any enough small positive $\epsilon$. Then, since $T^{*}$ is the optimal strategy we have that

$$
v\left(T^{*}\right) \geq v\left(T^{\epsilon, k}\right)
$$

Thus,

$$
\begin{aligned}
& \pi_{k} \ln \left(1+\frac{T_{k}^{*}}{N_{k}^{0}}\right)+\pi_{m} \ln \left(1+\frac{T_{m}^{*}}{N_{m}^{0}}\right) \\
& \geq \pi_{k} \ln \left(1+\frac{T_{k}^{*}-\epsilon / \pi_{k}}{N_{k}^{0}}\right)+\pi_{m} \ln \left(1+\frac{T_{m}^{*}+\epsilon / \pi_{m}}{N_{m}^{0}}\right) .
\end{aligned}
$$

So, putting $\epsilon \rightarrow 0$ we have that

$$
\frac{1}{T_{m}^{*}+N_{m}^{0}} \leq \frac{1}{T_{k}^{*}+N_{k}^{0}} \text { for any } m \neq k .
$$

Thus, there is a positive $\omega$ such that

$$
\frac{1}{T_{i}^{*}+N_{i}^{0}} \quad \begin{cases}=\omega, & \text { for } T_{i}^{*}>0, \\ \leq \omega, & \text { for } T_{i}^{*}=0 .\end{cases}
$$

So, the optimal strategy $T^{*}$ is of the form

$$
T(\omega)=\left(T_{1}(\omega), \ldots, T_{n}(\omega)\right)
$$

where

$$
T_{i}(\omega)=\left[1 / \omega-N_{i}^{0}\right]_{+} \text {for } i \in[1, n] .
$$


Reminding that the optimal strategy is non-negative vector satisfying the condition $\sum_{i=1}^{n} \pi_{i} T_{i}^{*}=\bar{T}$ we obtain that $\omega$ has to be found as a solution of the equation $H_{T}(\omega)=\bar{T}$. It is clear that $H_{T}(0+)=+\infty, H_{T}(\cdot)$ is continuous in $(0, \infty)$, $H_{T}(\omega)=0$ for $\omega \in\left[\max _{i}\left(1 / N_{i}^{0}\right), \infty\right)$ and $H_{T}(\cdot)$ is strictly decreasing in $\left(0, \max _{i}\left(1 / N_{i}^{0}\right)\right)$. Thus, there is unique positive $\omega$ such that $H_{T}(\omega)=\bar{T}$. This completes the proof of Theorem 1.

Proof of Theorem 2. First note $H(\omega)=0$ for $\omega \geq 1 / N_{1}^{0}$, $H(\omega)$ is strictly positive and decreasing in $\left(0,1 / N_{1}^{0}\right)$.

Let $k \in[1, n]$ be such that

$$
\frac{1}{N_{k}^{0}}>\omega^{*} \geq \frac{1}{N_{k+1}^{0}}
$$

where $N_{n+1}^{0}=\infty$.

Then, $\left[1 / \omega^{*}-N_{i}^{0}\right]_{+}=1 / \omega^{*}-N_{i}^{0}$ for $i \in[1, k]$ and $\left[1 / \omega^{*}-N_{i}^{0}\right]_{+}=0 i \in[k+1, n]$. So,

$$
H\left(\omega^{*}\right)=\sum_{i=1}^{k} \pi_{i}\left(1 / \omega^{*}-N_{i}^{0}\right) .
$$

Since $H\left(\omega^{*}\right)=\bar{T}$ we have that

$$
\omega^{*}=\frac{\sum_{i=1}^{k} \pi_{i}}{\bar{T}+\sum_{i=1}^{k} \pi_{i} N_{i}^{0}} .
$$

Because $H$ is strictly decreasing on $\left(0,1 / N_{1}^{0}\right)$ we can find $k$ from the following condition

$$
H\left(1 / N_{k}^{0}\right)<\bar{T} \leq H\left(1 / N_{k+1}^{0}\right) .
$$

Since

$$
\sum_{i=1}^{k} \pi_{i}\left(N_{k+1}^{0}-N_{i}^{0}\right)=\sum_{i=1}^{k+1} \pi_{i}\left(N_{k+1}^{0}-N_{i}^{0}\right),
$$

the integer $k$ can be found from the following equivalent condition

$$
\varphi_{k}<\bar{T} \leq \varphi_{k+1}
$$

where

$$
\varphi_{t}=\sum_{i=1}^{t} \pi_{i}\left(N_{t}^{0}-N_{i}^{0}\right) \text { for } t \in[1, n] .
$$

Therefore, Theorem 1, (19) and (20) imply Theorem 2.

Proof of Theorem 3. The Lagrangian corresponding to minimization of $-v^{j}$ subject to the constraint (4) and nonnegativity constraints on $T_{i}^{j}$ is given by

$$
\begin{gathered}
L^{j}=-\sum_{k=1}^{n} \pi_{k} \ln \left(1+\frac{T_{k}^{j}}{g T_{k}^{m}+N_{k}^{0}}\right)+\omega^{j}\left(\sum_{k=1}^{n} \pi_{k} T_{k}^{j}-\bar{T}^{j}\right) \\
+\nu_{i}^{j}\left(-T_{i}^{j}\right),
\end{gathered}
$$

with $m \neq j$. Differentiating the Lagrangian with respect to $T_{i}^{j}$ and equating the derivative to zero, we obtain

$$
\frac{1}{g T_{i}^{m}+T_{i}^{j}+N_{i}^{0}}+\frac{\nu_{i}^{j}}{\pi_{i}}=\omega^{j} .
$$

Now, using the complimentary slackness condition $\nu_{i}^{j} T_{i}^{j}=0$, we obtain condition (5). Since the left hand side of equation (21) is positive, the Lagrange multiplier $\omega^{j}$ is positive as well.

Proof of Lemma 1. (i) follows directly from (5) where $T_{i}^{1 *}=T_{i}^{2 *}=0$.

(ii) Let $T_{i}^{1 *}>0$ and $T_{i}^{2 *}=0$. Then by (5) we have that

$$
\frac{1}{T_{i}^{1 *}+N_{i}^{0}}=\omega^{1}
$$

Thus, $\frac{1}{\omega^{1}}>N_{i}^{0}$ and $T_{i}^{1 *}=\frac{1}{\omega^{1}}-N_{i}^{0}$. Then, by (5) we have that

$$
\omega^{2} \geq \frac{1}{g T_{i}^{1 *}+N_{i}^{0}}=\frac{1}{g\left(\frac{1}{\omega^{1}}-N_{i}^{0}\right)+N_{i}^{0}} .
$$

Thus,

$$
g\left(\frac{1}{\omega^{1}}-N_{i}^{0}\right) \geq \frac{1}{\omega^{2}}-N_{i}^{0}
$$

and the result follows.

(iii) can be proved similarly to (ii).

(iv) Let $T_{i}^{1 *}>0$ and $T_{i}^{2 *}>0$. Then, by (5)we have that $\frac{1}{\omega^{1}}>N_{i}^{0}$ and $\frac{1}{\omega^{2}}>N_{i}^{0}$. Also, by (5) we have that $T_{i}^{1 *}$ and $T_{i}^{2 *}$ are given by (6). Then, since $T_{i}^{1 *}>0$ and $T_{i}^{2 *}>0$ we have that $i \in I_{11}^{\prime}\left(\omega^{1}, \omega^{2}\right)$. This completes the proof of the lemma.

Proof of Lemma 2. Let, for example, $\omega^{1}<\omega^{2}$. It is clear that $I_{00}^{\prime}\left(\omega^{1}, \omega^{2}\right)=I_{00}\left(\omega^{1}, \omega^{2}\right)$.

Let $i \in I_{10}^{\prime}\left(\omega^{1}, \omega^{2}\right)$. Then either $1 / \omega^{2} \leq N_{i}^{0}<1 / \omega^{1}$ or $\frac{1 / \omega^{2}-g / \mu}{1-g} \leq N_{i}^{0}<\min \left\{1 / \omega^{1}, 1 / \omega^{2}\right\}$. Since $\omega^{1}<\omega^{2}$, $\min \left\{1 / \omega^{1}, 1 / \omega^{2}\right\}=1 / \omega^{2}$ and $\frac{1 / \omega^{2}-g / \omega^{1}}{1-g}<1 / \omega^{2}$. Thus, $I_{10}^{\prime}\left(\omega^{1}, \omega^{2}\right)=I_{10}\left(\omega^{1}, \omega^{2}\right)$.

Let $i \in I_{01}^{\prime}\left(\omega^{1}, \omega^{2}\right)$. Then either $1 / \omega^{1} \leq N_{i}^{0}<1 / \omega^{2}$ or $\frac{1 / \omega^{1}-g / \omega^{2}}{1-g} \leq N_{i}^{0}<\min \left\{1 / \omega^{1}, 1 / \omega^{2}\right\}$. Since $\omega^{1}<$ $\omega^{2}$, then $\frac{1 / \omega^{1}-g / \omega^{2}}{1-g}>1 / \omega^{2}$. So, $I_{01}^{\prime}\left(\omega^{1}, \omega^{2}\right)=\emptyset=$ $I_{01}\left(\omega^{1}, \omega^{2}\right)$.

Let $i \in I_{11}^{\prime}\left(\omega^{1}, \omega^{2}\right)$. Then

$N_{i}^{0}<\min \left\{\frac{1 / \omega^{1}-g / \omega^{2}}{1-g}, \frac{1 / \omega^{2}-g / \omega^{1}}{1-g}\right\}=\frac{1 / \omega^{2}-g / \omega^{1}}{1-g}$.

Thus, $I_{11}^{\prime}\left(\omega^{1}, \omega^{2}\right)=I_{11}\left(\omega^{1}, \omega^{2}\right)$. This completes the proof of Lemma 2 .

Proof of Corollary 1. Assume that $\omega^{1} \geq \omega^{2}$. Then $1 / \omega^{1}-$ $g / \omega^{2} \leq 1 / \omega^{2}-g / \omega^{1}$. Thus, $T_{i}^{1}\left(\omega^{1}, \omega^{2}\right) \leq T_{i}^{2}\left(\omega^{1}, \omega^{2}\right)$ for $i \in[1, n]$. So,

$$
\bar{T}^{1}=\sum_{i=1}^{n} \pi_{i} T_{i}^{1}\left(\omega^{1}, \omega^{2}\right) \leq \sum_{i=1}^{n} \pi_{i} T_{i}^{2}\left(\omega^{1}, \omega^{2}\right)=\bar{T}^{2} .
$$

This contradiction completes the proof of Corollary 1.

Proof of Proposition 1. Suppose that $\left(T^{1 *}, T^{2 *}\right)$ be a Nash equilibrium. Then, similarly to Lemma 1 , we have to consider three cases (i)-(iii) where at least one of components of the vector $\left(T^{1 *}, T^{2 *}\right)$ is positive. 
(i) Let $T_{i}^{1 *}>0$ and $T_{i}^{2 *}=0$. Then, by (5), we have that

$$
\frac{1}{T_{i}^{1 *}+N_{i}^{0}}=\omega^{1} \text {. }
$$

Thus,

$$
\frac{1}{\omega^{1}}>N_{i}^{0} \text { and } T_{i}^{1 *}=\frac{1}{\omega^{1}}-N_{i}^{0} .
$$

Then, by (5)

$$
\omega^{2} \geq \frac{1}{T_{i}^{1 *}+N_{i}^{0}}=\frac{1}{\frac{1}{\omega^{1}}-N_{i}^{0}+N_{i}^{0}}=\omega^{1} .
$$

(ii) Let $T_{i}^{2 *}>0$ and $T_{i}^{1 *}=0$. Then, similarly to (ii), we have that

$$
T_{i}^{2 *}=\frac{1}{\omega^{2}}-N_{i}^{0}
$$

and

$$
\frac{1}{\omega^{2}}>N_{i}^{0}, \quad \omega^{1} \geq \omega^{2}
$$

(iii) Let $T_{i}^{1 *}>0$ and $T_{i}^{2 *}>0$. Then, by (5), have that

$$
\frac{1}{T_{i}^{1 *}+T_{i}^{2 *}+N_{i}^{0}}=\omega^{1}=\omega^{2} .
$$

Assume that $\omega^{1}>\omega^{2}$ then (i) does not hold, so $T_{i}^{1 *}=0$ for each $i$ which contradicts to (4). Similarly, the case $\omega^{1}<$ $\omega^{2}$ cannot hold.

Thus, $\omega^{1}=\omega^{2}=\omega$. So, $T_{i}^{1 *}$ and $T_{i}^{2 *}, i \in[1, n]$ have to be any non-negative such that

$$
T_{i}^{1 *}+T_{i}^{2 *}=\left[1 / \omega-N_{i}^{0}\right]_{+}
$$

and

$$
\sum_{i=1}^{n} \pi_{i} T_{i}^{1 *}=\bar{T}^{1}, \sum_{i=1}^{n} \pi_{i} T_{i}^{2 *}=\bar{T}^{2},
$$

where $\omega$ is the unique positive root of the equation

$$
\sum_{i=1}^{n} \pi_{i}\left[1 / \omega-N_{i}^{0}\right]_{+}=\bar{T}^{1}+\bar{T}^{2} .
$$

It is clear that there is a continuum of such strategies. For example if $\left(T^{1 *}, T^{2 *}\right)$ is the one of them, and let $T_{k}^{1 *}, T_{k}^{2 *}>0$ and $T_{m}^{1 *}, T_{m}^{2 *}>0$ for some $k$ and $m$. Then, it is clear that the following strategies for any enough small positive $\epsilon$ are also optimal:

$$
\begin{gathered}
\tilde{T}_{i}^{1 *}= \begin{cases}T_{i}^{1 *} & \text { for } i \neq k, m, \\
T_{i}^{1 *}+\epsilon & \text { for } i=k, \\
T_{i}^{1 *}-\epsilon \pi_{k} / \pi_{m} & \text { for } i=m,\end{cases} \\
\tilde{T}_{i}^{2 *}= \begin{cases}T_{i}^{2 *} & \text { for } i \neq k, m, \\
T_{i}^{2 *}-\epsilon & \text { for } i=k, \\
T_{i}^{2 *}+\epsilon \pi_{k} / \pi_{m} & \text { for } i=m .\end{cases}
\end{gathered}
$$

This completes the proof of Proposition 1.

\section{REFERENCES}

[1] E. Altman, K. Avrachenkov, and A.

Garnaev,"Jamming game in wireless networks with transmission cost". Lecture Notes in Computer Science, v.4465, pp.1-12, 2007.
[2] E. Altman, K. Avrachenkov, G. Miller and B. Prabhu, "Discrete power control: cooperative and non-cooperative optimization", in Proceedings of IEEE INFOCOM 200\%. An extended version is available as INRIA Research Report no.5818.

[3] S.T. Chung and J.M. Cioffi, "Rate and power control in a two-user multicarrier channel with no coordination: the optimal scheme vs. suboptimal methods", in Proceedings of IEEE VTC'02-Fall, v.3, pp.1744-1748, 2002.

[4] T. Cover and J. Thomas, Elements of Information Theory, Wiley, 1991.

[5] W. R. Heinzelman, A. Chandrakasan, and H. Balakrishnan, "Energy-efficient communication protocol for wireless microsensor networks," in Proc. of the 33rd Annual Hawaii International Conference on System Sciences, v.2, Jan. 2000.

[6] A.J. Goldsmith and P.P. Varaiya, "Capacity of fading channels with channel side information", IEEE Trans. Information Theory, v.43(6), pp.1986-1992, 1997.

[7] T. J. Kwon and M. Gerla, "Clustering with power control," in Proc. IEEE Military Communications Conference (MILCOM'99), v.2, Atlantic City, NJ, USA, 1999, pp.1424-1428.

[8] L. Lai and H. El Gamal, "The water-filling game in fading multiple access channels", submitted to IEEE Trans. Information Theory, November 2005, available at http://www.ece.osu.edu/ helgamal/.

[9] C. R. Lin and M. Gerla, "Adaptive clustering for mobile wireless networks," IEEE JSAC, v.15, no.7, pp.1265-1275, 1997.

[10] Z.-Q. Luo and J.-S. Pang, "Analysis of iterative waterfilling algorithm for multiuser power control in digital subscriber lines", EURASIP Journal on Applied Signal Processing, 2006.

[11] O. Popescu and C. Rose, "Water filling may not good neighbors make", in Proceedings of GLOBECOM 2003, v.3, pp.1766-1770, 2003.

[12] D.C. Popescu, O. Popescu and C. Rose, "Interference avoidance versus iterative water filling in multiaccess vector channels", in Proceedings of IEEE VTC 2004 Fall, v.3, pp.2058-2062, 2004.

[13] D. Tse and P. Viswanath, Fundamentals of Wireless Communication, Cambridge University Press, 2005.

[14] W. Yu, Competition and cooperation in multi-user communication environements, $\mathrm{PhD}$ Thesis, Stanford University, June 2002.

[15] W. Yu, G. Ginis and J.M. Cioffi, "Distributed multiuser power control for digital subscriber lines", IEEE JSAC, v.20, pp.1105-1115, 2002. 